\title{
BALÓN DE BAKRI EN HEMORRAGIA POSPARTO EN MEDELLÍN (COLOMBIA): REPORTE DE CASO Y REVISIÓN DE LA LITERATURA
}

\author{
A case report and literature review regarding \\ the Bakri balloon in postpartum hemorrhage in \\ Medellín (Colombia)
}

Érika Fonseca-Chimá, M.D.*

Recibido: enero 27/10 - Aceptado: noviembre 8/10

\section{RESUMEN}

Objetivo: presentar un caso de atonía uterina que no respondió al manejo inicial y en el que se utilizó el balón de Bakri con control satisfactorio de la hemorragia. Se revisó la literatura respecto a la técnica quirúrgica de su inserción, las cointervenciones a administrar y las complicaciones relacionadas con su uso.

Materiales y métodos: se presentó el caso y se realizó una búsqueda bibliográfica en las bases de datos electrónicas de MEDLINE/Pubmed, Lilacs, Scielo, Ovid, Elsevier, EBSCO y Cochrane a partir de los términos "taponamiento con balón" y "hemorragia posparto”. Para ello se buscaron artículos de revisión, series de caso, reportes de caso y experimentos clínicos publicados entre 1980 y 2010.

Conclusiones: el balón de Bakri ha demostrado ser una opción en el protocolo de manejo de las pacientes con Hemorragia Posparto (HPP), ya que constituye un método no invasivo y con pocas complicaciones.

Palabras clave: taponamiento con balón, hemorragia posparto.

\section{SUMMARY}

Objective: presenting a case of uterine atony which did not respond to initial management; the Bakri

* Especialista en Ginecología y Obstetricia, Universidad de Antioquia, Medellín (Colombia).Correo electrónico: erfonseca@hotmail.com balloon was then used with satisfactory control of postpartum hemorrhage (PPH). The literature regarding the surgical technique for its insertion, the co-interventions to be administered and the complications related to its use were reviewed.

Materials and methods: the case was presented and a bibliographic search was made of the MEDLINE/ PubMed, Lilacs, Scielo, Ovid, Elsevier, EBSCO and Cochrane databases based on the terms "Balloon tamponade", "postpartum hemorrhage." Review articles, case series, case reports and clinical experiments were sought which had been published from 1980 to 2010 .

Conclusions: Bakri balloon for managing PPH has been shown to be an option in the protocol for managing these patients, as it is a non-invasive method and has few complications.

Key words: balloon tamponade, postpartum hemorrhage.

\section{INTRODUCCIÓN}

La HPP es la principal complicación del parto, siendo responsable del 25\% de las muertes maternas a nivel mundial. ${ }^{1}$ En Colombia, en el año 2006, la hemorragia obstétrica fue la segunda causa de muerte materna después de los fenómenos hipertensivos. ${ }^{2}$ La atonía uterina se encuentra entre las principales causas de hemorragia posparto. En el manejo de la atonía uterina, la primera opción terapéutica consiste en 
instaurar un manejo con medicamentos uterotónicos y reemplazar la volemia perdida con cristaloides o hemoderivados. ${ }^{3}$ Si no se presenta una respuesta adecuada, el algoritmo de manejo apunta hacia opciones quirúrgicas tales como técnica de B-Lynch, ${ }^{3-13}$ ligadura de arterias uterinas ${ }^{14-16}$ e histerectomía ${ }^{17}$ en última instancia, la cual se reserva para cuando las medidas anteriores fallan. Este último procedimiento está relacionado con complicaciones serias tales como mayor pérdida de sangre, mayor permanencia hospitalaria, mayor riesgo de lesión visceral gastrointestinal y de vías urinarias e inconvenientes de fertilidad en el futuro, ${ }^{18-19}$ de allí la importancia de buscar otras opciones terapéuticas no invasivas y menos mórbidas para tratar este tipo de pacientes, tal como es el caso del taponamiento uterino. ${ }^{20-21}$

Se han descrito varios métodos compresivos. Inicialmente se realizaba empaquetamiento uterino, utilizando gasas estériles y con el cual se obtenía un control inmediato del sangrado en el 97\% de los casos, aunque este cayó en desuso gradualmente por sus frecuentes efectos adversos tales como trauma e infecciones. ${ }^{22}$ Más recientemente el taponamiento uterino se ha realizado con balones, bien sea de silicona o de látex, que se insuflan con solución salina para ejercer una presión hidrostática sobre el útero. ${ }^{23}$ Se han reportado varios tipos de balones en el manejo de la HPP: Sengstaken-Blakemore, Rusch y catéter condón. ${ }^{23}$ Recientemente, se propuso el balón de Bakri (BB) para el manejo de la hemorragia obstétrica, siendo considerado un método no invasivo, rápido y seguro para tratar la hemorragia posparto secundaria a atonía uterina. ${ }^{24-27}$ En este reporte, se presenta un caso de atonía uterina manejada con el balón de Bakri con el objetivo de revisar la técnica quirúrgica para su colocación, las cointervenciones y las complicaciones relacionadas con su uso.

En este reporte de caso se tuvieron en cuenta todas las consideraciones éticas para la investigación en humanos, consignadas en la resolución 8430 de 1993 del Ministerio de Salud, y se mantuvo en todo momento la confidencialidad de la paciente.

\section{REPORTE DEL CASO}

Paciente de 26 años, ama de casa, a quien se le realizó cesárea por macrosomía fetal sin complicaciones. Dos horas después presentó atonía uterina severa que fue manejada con oxitocina $20 \mathrm{U}$ IV, methergina $0,4 \mathrm{mg}$ IM y misoprostol $1.000 \mu \mathrm{g}$ intrarectal, sin obtener respuesta. Posteriormente, se adicionó carbetocina $100 \mathrm{mg}$ IV, sin presentar mejoría, y se incluyó 3000 cc de cristaloides y 2 U de glóbulos rojos empacados. Se decidió entonces colocar balón de Bakri que se infló con 500 cc y se verificó con ecografía. Esta mostró sangrado que continuaba saliendo a través del lumen aunque ya en menor cantidad. Se dejó gasa en vagina y se trasladó a la paciente a la unidad de alta dependencia obstétrica para monitorización continua con antibióticos profilácticos y oxitocina, obteniendo estabilización y una disminución importante del sangrado, por lo que se decidió desinflar el balón 24 horas después a $50 \mathrm{cc} /$ hora, manteniendo la infusión de oxitocina.

\section{MATERIALES Y MÉTODOS}

Se realizó una búsqueda bibliográfica en las bases de datos electrónicas de MEDLINE/Pubmed, Lilacs, Scielo, Ovid, Elsevier, EBSCO y Cochrane a partir de los términos "taponamiento con balón" y "hemorragia posparto”. Para ello se buscaron artículos de revisión, series de casos, reportes de casos y experimentos clínicos publicados entre 1980 y 2010.

\section{DISCUSIÓN}

El balón de Bakri es un recurso que se tiene a disposición en el manejo de pacientes de este tipo en las que la fertilidad futura es importante. Está indicado usualmente en aquellas pacientes que no responden adecuadamente al manejo farmacológico con uterotónicos y en quienes se quiere llevar a cabo un manejo conservador no quirúrgico, ${ }^{26}$ principalmente en casos de hemorragia posparto no traumática y en los que se han descartado restos placentarios intrauterinos. ${ }^{28}$

Bakri inicialmente describió el balón intrauterino en el manejo de la hemorragia secundaria a 
placenta previa ácreta durante una cesárea con o sin ligadura bilateral de arterias hipogástricas. ${ }^{29}$

Vittala y sus colegas reportaron 15 casos con hemorragia posparto posterior a parto vaginal y cesárea y en quienes se colocó el balón de Bakri cuando el manejo médico no tuvo éxito. En términos generales, el taponamiento fue efectivo en el $80 \%$ de los casos. También informaron que la cantidad de líquido insuflado fue mayor en pacientes multíparas y posterior a embarazo gemelar, y menor en las pacientes primíparas y posterior a cesárea. ${ }^{26}$

\section{Técnica quirúrgica y cointervenciones}

El balón de Bakri SOS (Surgical Obstetric Silicone, por sus siglas en inglés) está hecho de silicona, mide $58 \mathrm{~cm}$ de largo, tiene doble luz y una capacidad máxima de hasta $800 \mathrm{ml}$. Sin embargo, se recomienda que se insufle con 250 a $500 \mathrm{ml}$, dependiendo del tamaño y la capacidad del útero. ${ }^{25} \mathrm{~A}$ diferencia de otros balones, como el balón urinario de Rusch y el catéter condón, el balón de Bakri tiene un canal de drenaje en el extremo de la sonda que permite evacuar la cavidad uterina, evaluar la efectividad y medir el sangrado persistente de la paciente. Además, como el extremo de la sonda es corto, existe mayor contacto entre la superficie distal del balón y el fondo uterino, permitiendo un mayor efecto hemostático, lo cual no es posible cuando se utiliza el balón de Sengstaken-Blakemore.

Si posterior a un parto vaginal se decide colocar el balón de Bakri, este se introduce transvaginalmente usando unas pinzas de cuello para sostener el cérvix y guiándolo hasta el fondo uterino con otras pinzas. Este paso puede realizarse a ciegas, aunque también se puede llevar a cabo bajo visión ecográfica. En los casos en que el cérvix está dilatado, debe realizarse empaquetamiento vaginal una vez que el balón ha sido insuflado para evitar desplazar el balón hacia la vagina. Posteriormente, debe confirmarse su correcta ubicación a través de ecografía. ${ }^{26}$

Si se trata de una paciente a la que se le ha realizado cesárea, entonces se coloca el balón por vía abdominal y se introduce dentro de la cavidad uterina a través de histerorrafia, pasando retrógradamente la porción distal de la sonda a través del cérvix y hacia la vagina. Finalmente, se sutura el útero de forma convencional y se insufla el balón con solución salina. Otro abordaje alternativo consiste en cerrar el útero primero e insertar el balón desde la vagina. ${ }^{26}$ Una vez colocado el balón, se hace un test de taponamiento que consiste en llenar el balón hasta que el sangrado sea controlado. ${ }^{30,31}$ El test se considera positivo si el sangrado es controlado luego de la insuflación del balón y negativo en caso de persistir el sangrado, sugiriendo la realización de otro tipo de manejo quirúrgico más agresivo. ${ }^{32}$ Por otra parte, se ha reportado ubicación del balón en el segmento uterino cercano al istmo, comprimiendo las arterias uterinas. Esta posición podría considerarse errónea por algunos. Sin embargo, los autores señalan que el sangrado intratable fue controlado exitosamente y consideran que este podría ser otro mecanismo de acción del balón de taponamiento. ${ }^{33}$

Mientras el balón permanezca en la cavidad uterina, la oxitocina debe continuarse en infusión continua durante 24 horas. ${ }^{29,31,34-36}$ La carbetocina, un análogo sintético de la oxitocina, tiene una vida media más larga (4 a 10 veces comparada con la oxitocina), ${ }^{37}$ por lo que puede ser el medicamento de elección para conseguir contracción por largo tiempo cuando se coloca balón intrauterino. También se debe dejar sonda vesical para vigilar el gasto urinario.

Al usar el balón, es importante reducir el riesgo de infección iatrogénica por bacterias vaginales que producen contaminación hacia la cavidad uterina. Varias publicaciones sugieren una cefalosporina como el antibiótico indicado. La duración de este puede ser profiláctica (dosis única) o continua por 24 hasta 48 horas mientras el balón permanezca en la cavidad uterina. ${ }^{38}$

El dolor causado por el útero distendido puede aliviarse con medicamentos tipo opioides o con anestesia continua en los casos en que el balón se coloca posterior a una cesárea. En caso de persistencia del 
dolor a pesar de manejo analgésico, otra opción consiste en reducir un poco la insuflación del balón, teniendo en cuenta que debe haber un equilibrio entre el efecto hemostático y el alivio del dolor. ${ }^{26}$

En la mayoría de los reportes se ha retirado el balón en 24 horas, con una tasa de desinflado que varía desde $20 \mathrm{ml} / \mathrm{h}$ hasta la mitad del volumen del balón en 12 horas. ${ }^{35,38}$ No obstante, la duración del balón intrauterino puede estar supeditado a la clínica de la paciente en los casos en que el sangrado continúa. ${ }^{38}$

\section{Complicaciones}

En general, se han atribuido pocos efectos adversos relacionados con el uso del balón intrauterino. Un reporte de caso menciona endometritis posterior al uso del balón de Rusch a pesar de recibir antibióticos por 24 horas. Es importante aclarar que esta paciente tuvo un trabajo de parto prolongado y un parto instrumentado, los cuales pueden tener una relación causal más probable. ${ }^{38}$ Otro estudio reportó una paciente que presentó fiebre y que respondió a antibióticos después de usar catéter condón. ${ }^{39}$

La opción de sobreinflar el balón en el útero para prevenir el desplazamiento puede ser perjudicial y causar otros problemas. La distensión del útero, por ejemplo, puede causar dolor importante a la paciente. ${ }^{38}$ Otra complicación teórica podría ser la ruptura uterina, aunque no se ha publicado ningún caso al respecto. Pocos estudios han reportado fallas o dificultades en el uso de balones, tales como dificultad en la inserción por miomas uterinos, daño inadvertido del balón mientras se prepara para ser usado o incapacidad para colocar el balón debido a la presencia de una sutura tipo B-lynch. $^{30}$

Existen complicaciones potenciales con el uso de balón intrauterino, pero aún no se ha reportado ninguna. Estas pueden incluir ulceración del útero y de la vagina por la presión ejercida por el balón, especialmente por uso prolongado, ruptura uterina por sobredistensión y perforación uterina durante la inserción. ${ }^{30}$

\section{CONCLUSIONES}

El balón de Bakri surge como una alternativa a considerar en el manejo de la paciente con hemorragia obstétrica cuando no hay una buena respuesta al manejo convencional. No obstante, este no es un método que se pueda utilizar de forma aislada.

\section{AGRADECIMIENTOS}

A la Clínica del Prado en Medellín (Colombia) por permitirme tener experiencias como el caso antes reportado, que aportaron mucho en mi vida profesional.

\section{REFERENCIAS}

1. World Health Organization. The World Report 2005. Attending to 136 million births, every year: make every mother and child count. Geneva: WHO; 2005. p. 62-63.

2. Departamento Administrativo Nacional de Estadística, DANE. Estadísticas vitales. Bogotá D.C. Visitado en 2010 Sep 29. Disponible en: http://www.dane.gov.co/ index.php?option $={ }_{\text {com_content } \& \text { task }}=$ category\&se ctionid $=168$ id $=36 \&$ Itemid $=148$

3. Vélez-Álvarez G, Agudelo-Jaramillo B, Gómez-Dávila G, Zuleta-Tobón JJ. Código rojo: guía para el manejo de la hemorragia obstétrica. Rev Colomb Obstet Ginecol 2009;60:34-48.

4. Mousa HA, Alfirevic Z. Major pospartum hemorrhage: survey of maternity units in the United Kindom. Acta Obstet Gynecol Scand 2002;81:727-30.

5. Tamizian O, Arulkumaran S. The surgical management of postpartum haemorrhage. Curr Opin Obstet Gynecol 2001;13:127-31.

6. Doumouchtsis SK, Papageorghiou AT, Arulkumaran S. Systematic review of conservative management of postpartum hemorrhage: what to do when medical treatment fails. Obstet Gynecol Surv 2007;62:540-7.

7. Knight M, UKOSS. Peripartum hysterectomy in the UK: management and outcomes of the associated haemorrhage. BJOG 2007;114:1380-7.

8. Ramsbotham PH. The principles and practice of obstetrical medicine and surgery. Philadelphia PA: Blanchard and Lea; 1955. 
9. Allahdin S, Aird C, Danielian P. B-Lynch sutures for major primary postpartum haemorrhage at caesarean section. J Obstet Gynaecol 2006;26:639-42.

10. Danso D, Reginald P. Combined B-Lynch suture with intrauterine balloon catheter triumphs over massive postpartum haemorrhage. BJOG 2002;109:963.

11. Ferguson JE, Bourgeois FJ, Underwood PB. B-Lynch suture for postpartum hemorrhage. Obstet Gynecol 2000;95:1020-2.

12. Habek D, Kulas T, Bobic-Vukovic M, Selthofer R, Vujić B, Ugljarević M. Successful of the B-Lynch compression suture in the management of massive postpartum hemorrhage: case reports and review. Arch Gynecol Obstet 2006;273:307-9.

13. Wohlmuth CT, Gumbs J, Quebral-Ivie J. B-Lynch suture: a case series. Int J Fertil Womens Med 2005;50:164-73.

14. O'Leary JL, O'Leary JA. Uterine artery ligation in the control of intractable postpartum hemorrhage. Am J Obstet Gynecol 1966;94:920-4.

15. O'Leary JL, O’Leary JA. Uterine artery ligation for control of postcesarean section hemorrhage.Obstet Gynecol 1974;43:849-53.

16. O'Leary JA. Uterine artery ligation in the control of postcesarean hemorrhage. J Reprod Med 1995;40:189-93.

17. American College of Obstetricians and Gynecologists. ACOG Practice Bulletin: Clinical Management Guidelines for Obstetrician-Gynecologists Number 76, October 2006: postpartum hemorrhage. Obstet Gynecol 2006;108:1039-47.

18. Kwee A, Bots ML, Visser GH, Bruinse HW. Emergency peripartum hysterectomy: a prospective study in The Netherlands. Eur J Obstet Gynecol Reprod Biol 2006;124:187-92.

19. Al-Sibai MH, Rahman J, Rahman MS, Butalack F. Emergency hysterectomy in obstetrics a review of 117 cases. Aust N Z J Obstet Gynaecol 1987;27:180-4.

20. Douglas LH. The passing of the pack. Bull Sch med Univ Md 1955;40:38-9.

21. Drucker M, Wallace RC. Uterine packing: a reappraisal. Mt Sinai J Med 1979;46:191-4.

22. Lester WM. Bartholomew RA. Colvin ED, Grimes WH Jr, Fish JS. Galloway WH. Reconsideration of the uterine pack in postpartum hemorrhage. Am J Obstet Gynecol 1965;93:321-9.

23. Georgiou C. Ballon tamponade in the management of postpartum hemorrhage: a review. BJOC 2009; 116:748-57.

24. Brace V, Kernaghan D, Penney G. Learning from adverse outcomes major obstetric hemorrhage in Scotland. BJOG 2007;114:1388-96.

25. Cook Medical. Enclosed instruction leaflet J-SOS 1106.

26. Vitthala S, Tsoumpou I, Kanjun A, Aziz N. Use of Bakri balloon in postpartum hemorrhage: a series of 15 cases. Aust N Z J Obstet Gynecol 2009;49:191-4.

27. Morales A, Insunza A, Latorre R. Manejo exitoso de la hemorragia posparto por acretismo placentario mediante taponamiento con balón de Bakri. Rev Chil Obstet Ginecol 2006;71(2):121-4.

28. Lalonde A, Daviss BA, Acosta A, Herschderfer K. Postpartum Hemorrhage today: ICM/FIGO iniative 2004-2006. Int J Gynecol Obstet 2006;94:243-53.

29 Bakri YN. Uterine tamponade-drain for hemorrhage secondary to placenta previa-accreta. Int J Gynecol Obstet 1992;37:302-3.

30. Dabelea V, Schultze PM, McDuffie RS. Intrauterine balloon tamponade in the management of postpartum hemorrhage. Am J Perinatol 2007;24:359-64.

31. Akhter S, Begum MR, Kabir J. Condom hydrostatic tamponade for massive postpartum hemorrhage. Int J Gynaecol Obstet 2005;90:134-5.

32. Condous GS, Arulkumarah S, Symonds I, Chapman R, Sinha A, Razvi K. The "tamponade test" in the management of massive postpartum hemorrhage. Obstet Gynecol 2003;101:767-72.

33. Cho Y, Rizvi C, Uppal T, Condous G. Ultrasonographic visualization of balloon placement for uterine tamponade in massive primary postpartum hemorrhage. Ultrasound Obstet Gynecol 2008;32:711-3.

34. Bakri YN, Amri A, Abdul Jabbar F. Tamponadeballoon for obstetrical bleeding. Int J Gynaecol Obstet 2001;74:139-42.

35. Katesmark M, Brown R, Raju KS. Successful use of a Sengstaken-Blakemore tube to control massive postpartum haemorrhage. Br J Obstet Gynaecol 1994;101:259-60. 
36. Johanson R, Kumar M, Obhrai M, Young P. Management of massive postpartum haemorrhage: use of a hydrostatic balloon catheter to avoid laparotomy. BJOC 2001;108:420-2.

37. Dansereau J, Joshi AK, Helewa ME, Doran TA, Lange IR, Luther ER, et al. Double-blind comparison of carbetocin versus oxytocin in prevention of uterine atony after cesarean section. Am J Obstet Gynecol 1999;180:670-6.
38. Keriakos R, Mukhopadhyay A. The use of the Rusch balloon for management of severe postpartum haemorrhage. J Obstet Gynaecol 2006;26:335-8.

39. Bagga R, Jain V, Sharma S, Suri V. Postpartum hemorrhage in two women with impaired coagulation successfully managed with condom catheter tamponade. Indian J Med Sci 2007; 61:157-8.

Conflicto de intereses: ninguno declarado. 